\title{
INDUSTRIALIZACIÓN PRIMARIA DEL AGUAJE (Mauritia flexuosa L. f.) EN IQUITOS (PERÚ)
}

\author{
Roberto Rojas Ruiz, Carlo F. Salazar Jarama, Charles Llerena Flores, Cléver \\ Rengifo Sias, Julio Ojanama Vásquez, Vanessa Muñoz Isuiza, Hilter Luque \\ Salinas, Jorge Solignac Ruiz, Dervin Torres Noriega, Flor de María Panduro \\ Ruiz*.
}

\section{RESUMEN}

En Iquitos (3 45’ 05" S y 73 14’ 40" O; 231648 hab.), capital del mayor departamento amazónico del Perú, se estudió la industrialización primaria de los frutos del aguaje (Mauritia flexuosa L.f). Esta es realizada por ocho chupeterías, una heladería y tres heladeros. Las chupeterías más antiguas funcionan desde 1970 y la heladería desde 1940. Además del aguaje, elaboran también chupetes de nueve frutas regionales.

El abastecimiento se realiza mediante proveedores fijos, libres y por compra directa en los mercados y puertos de la ciudad. El costo por saco de frutos varía de 7 a 60 nuevos soles en el año. Cada industria consume desde 2 hasta 20 sacos por día. El ecotipo mayoritariamente usado es el «amarillo o posheco»; en menor medida, se utiliza el «shambo». El precio del chupete varía desde 0,2 hasta 1,0 nuevos soles. Se exporta a otras ciudades como Pucallpa y Lima. La industria emplea hasta 189 vendedores a quienes les pagan el $40 \%$ del precio de venta.

El equipamiento básico de la industria es una máquina chupetera y congeladoras. Los insumos utilizados son «masa» de aguaje, leche, azúcar y vainilla. El tiempo máximo de congelación es de tres días. Por ello, el gran problema de esta industria es la conservación de la masa, pues esta se oxida en un día.

Los industriales esperan propuestas de la universidad local para mejorar el flujo operacional y la calidad del producto. Asimismo, son conscientes del daño que causa al medio ambiente la forma de extracción de los frutos.

Palabras clave: Aguaje, mauritia, burití, chupetería, industria aguajera, Amazonía.

* Círculo de Estudios e Investigación de Palmeras Amazónicas de la Facultad de Ingeniería Forestal de la Universidad Nacional de la Amazonía Peruana. 


\begin{abstract}
In Iquitos (3 $3^{\circ} 45^{\prime} 05^{\prime \prime} \mathrm{S}$ and $73^{\circ} 14^{\prime} 40^{\prime \prime} \mathrm{W}$; 231648 hab.) capital of the largest Amazonic Department of Peru, was studied the primary industrialization of the fruits of aguaje (Mauritia flexuosa L. f.); that is made by eight solid ice-cream stores, one ice-cream store, and three ices-cream dealers. Oldest solid ice-cream stores work since 1970 and the ice-cream stores works since 1940. Besides aguaje, they also process solid ice-cream of nine regional fruits.

The supplying is made by determined suppliers, free suppliers and by direct purchase in the markets and harbors of the city, the bag by coat of fruits varies of 7 to 60 nuevos soles during the year. Each industry uses from 2 to 20 coats per day, «yellow or posheco» is the main ecotype used and the lesser is «shambo»; the price of the solid ice-cream varies from 0,2 to 1 nuevo sol and are exported to other cities like Pucallpa and Lima; the industry uses up to 189 people as salesmen paying $40 \%$ of the sale price to them.

The basic equipment of the industry is an solid ice-cream machine and a freezer, the used ingredients are mass of aguaje, milk, sugar and vanilla; the maximum time of freezing are three days and the great problem is the conservation of the mass that oxidizes in a day.

The industrials are waiting the proposals of the local University to improve the operational flow and the quality of the product, also is conscious of the damage to the environment, by the form of extraction of the fruits.
\end{abstract}

Key Words: Aguaje, mauritia, burití, solid ice-cream store, industry of aguaje, Amazon.

\title{
1. INTRODUCCIÓN
}

El aguaje (Mauritia flexuosa L. f.), la palmera de mayor importancia económica en el Perú (Ruiz, 1991,1993; Padoch, 1992), ha sido muy poco estudiado y quizás hasta olvidado por las instituciones de investigación y desarrollo. Las mayores referencias sobre el uso económico de la especie son de investigadores e instituciones extranjeras (Granville, 1974; Heinen y Ruddle, 1974; NAS, 1975; Balick, 1979, 1982; Padoch, 1988 a,b, 1992; Henderson, 1995; Henderson, Galeano y Bernal, 1995).

El interés nacional por la especie se observa recién en estos últimos cinco años y, con mayor énfasis, en el año 2000. 
La importancia económica, social, industrial, ecológica y medicinal de los subproductos de esta palmera, en todos los países donde crece, es evidente, tal como puede comprobarse, por ejemplo, en el Perú, donde se calcula que 5000 familias están vinculadas con el comercio del aguaje. En Venezuela, las poblaciones indígenas usan la masa seca como pan (Braun, 1968); en Brasil, la mayor fuente alimenticia durante la época de inundaciones para las poblaciones ribereñas del Estado do Pará, se apoya en el aguaje (Hiraoka, 1999) y, en Ecuador, el aguaje juega un importante papel en la alimentación de las poblaciones nativas (Vickers, 1976).

En el Perú, los frutos son conocidos y usados en diferentes formas en las ciudades ubicadas en la región amazónica. Particularmente, en la ciudad de Iquitos, que sin lugar a dudas puede ser considerada como el mayor centro de consumo de aguaje en el mundo, se concentran los mayores volúmenes de consumo.

Por las razones expuestas y con la finalidad de contribuir a un mayor conocimiento del rol que desempeña el aguaje en la economía de la ciudad, se desarrolló este estudio. Los objetivos de este trabajo son: conocer las industrias de transformación del producto en cuestión, su proceso de transformación, el abastecimiento de materia prima, su comercialización y las expectativas y preocupaciones de los industriales.

\section{MATERIALES Y MÉTODOS}

El ámbito de estudio del presente trabajo se ubica al nordeste del Perú en la región natural denominada selva baja u omagua, en la ciudad de Iquitos, capital del departamento de Loreto. La ciudad se asienta en la margen izquierda del río Amazonas y está rodeada por los ríos Itaya y Nanay y por el lago Morona Cocha.

Las coordenadas geográficas de la ciudad son $3^{\circ} 45^{\prime} 05^{\prime}$ ' S y $73^{\circ} 14^{\prime} 40^{\prime \prime} \mathrm{O}$; se levanta 122,4 m sobre el nivel del mar a una distancia de 1928 millas de navegación hasta el Atlántico (datos ubicados en la plaza de armas de la ciudad y levantados por la Marina de Guerra del Perú).

La zona de estudio, en el nivel de biomasa, está comprendida en la Provincia Biogeográfica Amazónica Tropical; en el nivel de ecosistemas, predomina la zona de vida Bosque Húmedo Tropical (bh-T) y, de acuerdo con el mapa climático del Perú, el área se encuentra dentro del tipo de clima A(r)A' H4, que se caracteriza por ser muy lluvioso (Flores, Gómez y Kalliola, 1998). 
Los promedios climatológicos de la ciudad de Iquitos son: para las temperaturas media, máxima y mínima, 26,30 y $22{ }^{\circ} \mathrm{C}$, respectivamente; la precipitación mensual es de $257 \mathrm{~mm}$; la precipitación anual, de $3087 \mathrm{~mm}$ y la humedad relativa máxima y mínima de 95 y 74\%, respectivamente (Marengo, 1998). Se distinguen dos estaciones: una seca, desde junio hasta octubre, y otra lluviosa, desde noviembre hasta mayo.

La población de Iquitos es de 231648 habitantes y su densidad poblacional, de 51,64 $\mathrm{hab} / \mathrm{km}^{2}$, que es una cifra alta en comparación con la del departamento que es de 2,28 $\mathrm{hab} / \mathrm{km}^{2}$ (INEI, 1993). El acceso a la ciudad, desde el resto del Perú, se realiza por vía fluvial o aérea.

Para el levantamiento de la información, se llevaron a cabo los siguientes pasos:

a) Revisión de los archivos de la Municipalidad Provincial de Maynas y de la Municipalidad Distrital de Punchana para obtener la relación de industrias oficialmente registradas.

b) Ubicación de las direcciones de las industrias registradas en las municipalidades para comprobar su existencia.

c) Ubicación de otras chupeterías existentes y no registradas en las municipalidades con la finalidad de conocer el universo de las industrias.

d) Realización de encuestas estructuradas con entrevistas directas a los propietarios de las chupeterías y heladerías y a los heladeros de la ciudad.

e) Visita a las instalaciones de las industrias para conocer la infraestructura de los locales.

Con toda la información comprobada, se elaboraron tablas para facilitar la interpretación y el análisis de la misma.

\section{RESULTADOS Y DISCUSIÓN}

\subsection{Infraestructura de la industria de transformación industrial primaria del aguaje}

La transformación industrial primaria del fruto del aguaje, en la ciudad de Iquitos, es realizada por ocho chupeterías, una heladería y tres heladeros. Las tres chupeterías 
más antiguas tienen 30 años de funcionamiento y las cinco restantes se establecieron en la década del 90 (Tabla 1).

Se conoce la existencia de chupeterías en Iquitos desde hace más de 50 años. La no continuidad en el negocio se debe a diversas causas, pero, definitivamente, no es debida a la escasez de materia prima o a la baja rentabilidad del negocio.

La relación mostrada en la Tabla 1 solo indica la existencia actual de las chupeterías, lo cual no significa que antes y después de 1970 no hayan existido otras.

Las chupeterías y la heladería, a diferencia de los heladeros, también elaboran chupetes y helados de otras frutas regionales. Estas han incorporado hasta nueve de ellas en su producción; sin embargo, siempre están experimentando con nuevas frutas y solo continúan con la producción de aquellas que logran la aceptación del público consumidor.

Generalmente, la elaboración de chupetes de otras frutas se realiza cuando estas aparecen en el mercado local. Dicha elaboración no ocurre cuando la estación de producción de estas frutas acaba, mientras que solo el fruto del aguaje es constante durante el año.

Para la preparación de los chupetes, siempre se utilizan frutas frescas; sin embargo, también se fabrican chupetes cuyos insumos son solo esencias (fresa y chocolate), pero cuyos sabores gozan de la aceptación de la población.

La heladería La Favorita, establecida en 1940, continúa ofertando los helados de aguaje y de otras frutas regionales. Por su parte, los heladeros solo ofertan helados de aguaje. La diferencia entre las heladerías y los heladeros estriba en el lugar que ocupan y en la modalidad de venta del producto. Mientras que la heladería es un establecimiento fijo, el heladero es una persona que oferta el producto en un medio móvil y pequeño que diariamente se traslada al lugar de venta.

En Iquitos existían muchos heladeros, pero en la actualidad solo existen tres. Uno de ellos, que vende en la esquina de las calles Próspero y Napo, realiza este trabajo desde el año 1956 (durante 44 años), pero se estableció en su lugar actual solo desde hace 15 años. 
Tabla 1: Chupeterías, heladerías, heladeros, antigüedad y tipos de chupetes y helados en Iquitos.

\begin{tabular}{llcl}
\hline No $^{\circ}$ & Nombre de la chupetería & $\begin{array}{c}\text { Año de } \\
\text { establecimiento }\end{array}$ & Tipos de chupetes \\
\hline 1 & Shambo & 1970 & a, c, cc, g, h, p, u, v \\
2 & Oh qué rico & 1970 & a, c, f, u \\
3 & Las delicias & 1970 & a, c, f, u \\
4 & El buen sabor & 1977 & a, c, ch, f, u \\
5 & Celsa & 1991 & a, c, u \\
6 & Sevillano & 1995 & a, c, u \\
7 & Ivory & 1998 & a, c, f, u \\
8 & Saborelly & 2000 & a, c, f, m, u \\
\hline
\end{tabular}

Heladerías y heladeros

\begin{tabular}{llll}
\hline 1 & Heladería La Favorita & 1940 & $\mathrm{a}, \mathrm{c}, \mathrm{cc}, \mathrm{ch}, \mathrm{g}, \mathrm{u}, \mathrm{v}$ \\
\hline 1 & Esquina Próspero/Napo & 1985 & $\mathrm{a}$ \\
2 & En el colegio San Agustín & 1987 & $\mathrm{a}$ \\
3 & Esquina Próspero/Brasil & 1992 & $\mathrm{a}$ \\
\hline
\end{tabular}

a: aguaje (Mauritia flexuosa L. f.),

c: coco (Cocos nucifera L.),

cc: camu camu (Myrciaria dubia (H.B.K.) McVaugh),

ch: chocolate (Theobroma cacao L.),

f: fresa (Fraguaria sp.),

$\mathrm{g}$ : guayaba brasileña (Eugenia stipitata McVaugh), h: umari (Poraqueiba sericea Tul), $\mathrm{m}$ : mango (Mangifera indica $\mathrm{L}$ ), p: pijuayo (Bactris gasipaes H.B.K.), u: ungurahui (Oenocarpus bataua $\mathrm{M})$, v: ubos (Spondias mombin L).

\subsection{Abastecimiento de materia prima para la industria aguajera}

Según la Tabla 2, la forma cómo se abastecen las industrias de los frutos del aguaje se distribuye de la siguiente manera: el 50\% de las chupeterías se abastece a través de proveedores libres, es decir, de mayoristas medianos que recogen el producto y lo ofertan a las diferentes chupeterías. En este caso, los industriales tienen la ventaja de seleccionar el producto que pueden aceptar o rechazar, pero tienen la desventaja de no obtener los frutos con facilidad en épocas de escasez.

El 25\% de las chupeterías se abastece de proveedores fijos. Estas chupeterías tienen la ventaja de obtener el producto todo el año, pues el proveedor adquiere el compromiso de abastecerlas durante todo este periodo. El restante $25 \%$ adquiere el producto directamente del puerto de desembarque o de los mercados de la ciudad. 
Siete de las chupeterías y la heladería adquieren el producto en forma de fruto. Solo una se abastece de la materia prima en forma de masa diluida. Por su parte, los heladeros adquieren la materia prima en forma de masa en los mercados de Iquitos.

El precio del saco de aguaje (de 35 a $40 \mathrm{~kg}$ ) varía desde siete soles, en la época de mayor abundancia (entre julio y octubre), hasta 60 soles, en época de escasez. Entre los heladeros también se observa esta variación en el precio de la bolsa de masa de aguaje (de 600 a $700 \mathrm{~g}$ ), que es de uno a dos soles.

No están claramente establecidos los lugares que producen los frutos de mejor calidad. Las opiniones son variadas y, al parecer, la calidad está relacionada con las zonas de extracción de los proveedores. Sin embargo, en este estudio se confirma que las zonas de mayor extracción están ubicadas en los ríos Napo, Marañón y Ucayali, tal como lo informó Padoch (1992).

Las chupeterías, de acuerdo con su capacidad de producción, usan diariamente entre 2 y 18 sacos de aguaje. Son cuatro las chupeterías que procesan el $76 \%$ de los sacos usados y las cuatro restantes, el $24 \%$.

De esta información, se puede deducir que el consumo de frutos de aguaje, solo en la industria, es del orden de 73 a 97 sacos diarios que, proyectados mensualmente, arrojan un consumo de 2190 a 2910 sacos. Dicha cantidad, multiplicada por un peso promedio de $40 \mathrm{~kg} / \mathrm{saco}$, otorga un consumo de 87,6 a 116,4 tm/mes. Si este consumo se suma a los demás usos del fruto (como fruta y como refresco), que calculamos constituye un $50 \%$ más, fácilmente se puede proyectar que Iquitos consume entre 131,4 y 174,6 toneladas mensuales de frutos de aguaje.

Con esta información y estimándose que cada planta produce un promedio de $138 \mathrm{~kg}$ de frutos (Rojas, 1985), también se puede deducir que, en el departamento de Loreto, se cortan mensualmente 1000 palmeras de aguaje femeninas. Esta cifra, proyectada anualmente, arroja un total de 12000 palmeras, lo cual, desde todo punto de vista, está afectando seriamente los procesos ecológicos que ocurren en estas formaciones vegetales y que necesitan ser investigados. 
Tabla 2: Abastecimiento, precio, zona de mejor calidad de frutos y ecotipos usados.

\begin{tabular}{|c|c|c|c|c|c|c|c|}
\hline \multicolumn{8}{|c|}{ Chupeterías y chupeteros } \\
\hline $\mathrm{N}^{\circ}$ & $\begin{array}{l}\text { Forma de } \\
\text { abastecimiento }\end{array}$ & $\begin{array}{l}\text { Precio/saco } \\
\text { Soles }\end{array}$ & $\begin{array}{c}\text { Variación } \\
\text { en el año } \\
\text { Soles }\end{array}$ & $\begin{array}{l}\text { Zona de } \\
\text { mejor } \\
\text { calidad }\end{array}$ & $\begin{array}{c}\mathrm{N}^{\circ} \text { de sacos } \\
\text { usados } \\
\text { diariamente }\end{array}$ & $\begin{array}{c}\text { Ecotipo } \\
\text { usado }\end{array}$ & $\begin{array}{l}\text { Razón } \\
\text { para } \\
\text { preferir } \\
\text { el ecotipo }\end{array}$ \\
\hline 1 & Proveedor fijo & 40 (balde) & $40-60$ & - & $3-5$ & varios & - \\
\hline 2 & Proveedores & 10 & $10-50$ & Napo Marañón & $15-18$ & amarillo & da color \\
\hline 3 & Proveedores & 10 & $10-50$ & Napo Marañón & $15-18$ & amarillo & da color \\
\hline 4 & $\begin{array}{l}\text { Puertos y } \\
\text { mercados }\end{array}$ & 10 & $10-40$ & No sabe & 4 & varios & $\begin{array}{c}\text { solo } \\
\text { interesa } \\
\text { calidad }\end{array}$ \\
\hline 5 & Proveedores & $10-15$ & $10-60$ & $\begin{array}{l}\text { Saramuro, } \\
\text { Saramiriza, } \\
\text { Iq. - Nauta }\end{array}$ & $10-13$ & amarillo & da color \\
\hline 6 & $\begin{array}{l}\text { Puertos y } \\
\text { mercados }\end{array}$ & 7 & $7-50$ & Ucayali & 2 & amarillo & da color \\
\hline 7 & Proveedor fijo & 15 & $15-40$ & Napo & $5-8$ & shambo & más cremoso \\
\hline 8 & Proveedores & $10-15$ & $10-40$ & No sabe & 6 & shambo & da buen color \\
\hline \multicolumn{8}{|c|}{ Heladerías y heladeros } \\
\hline 1 & $\begin{array}{l}\text { Puertos y } \\
\text { mercados }\end{array}$ & 10 & $10-60$ & Ucayali & $10-20$ & shambo & más pulpa \\
\hline & Mercados & 1,5 bolsa & $1,5-2$ & Ucayali & 1 balde & amarillo & da color \\
\hline & Mercados & 1 bolsa & $1-2$ & No sabe & 1 balde & amarillo & da color \\
\hline 4 & Mercados & 1 bolsa & $1-2$ & No sabe & 1 balde & amarillo & da color \\
\hline
\end{tabular}

Saco: $1 \mathrm{saco}=35-40 \mathrm{~kg}$ de frutos que producen 38 bolsas de masa.

Balde: capacidad para 38 bolsas de masa de aguaje.

Bolsa: bolsas plásticas con capacidad de $0,25 \mathrm{~kg}$, pero la masa colocada allí (mesocarpo y cáscara) alcanza un peso promedio de $0,7 \mathrm{~kg}$.

Mes de referencia: octubre de 2000.

El ecotipo de aguaje generalmente usado es el «amarillo o posheco» porque, según los industriales, otorga y fija el color característico de los chupetes. Solo dos de las chupeterías prefieren el ecotipo «shambo», puesto que consideran que es de mejor calidad y tiene más crema. Sin embargo, para otros propietarios, este último ecotipo no es bueno para fabricar chupetes, pues consideran que contiene mucho aceite y que, al momento de congelar la dilución, este se acumula en un solo lugar y resta calidad al producto. 


\subsection{Comercialización de los productos industriales del aguaje}

Según la Tabla 3, la comercialización de los chupetes se realiza bajo tres modalidades:

a) A través de la fábrica o la chupetería. Todas las chupeterías venden sus productos directamente al público y al mismo precio que los chupeteros.

b) Por medio de los chupeteros. Se llama así a las personas de diferentes edades que ofertan el producto circulando por las calles de la ciudad. Los envases usados para transportar el producto son de tres tipos: cajas de technoport, cajas de espuma de vidrio y carritos manuales.

c) A través de bodegas: comercios que venden productos de primera necesidad. Entre sus productos, están los chupetes.

La temporada de mayores ventas es la estación seca, llamada verano (desde junio hasta octubre), que es coincidente con la estación de vaciante de los ríos amazónicos. Sin embargo, también se registran ventas altas durante las fiestas navideñas: año nuevo y todo feriado cívico en que se celebre algún acontecimiento importante.

Todas las chupeterías fabrican chupetes de 0,5 soles, pero cuatro de ellas (50\%) elaboran chupetes de $1 \mathrm{sol}$, a los que denominan «chupetes especiales». Estos tienen una mejor calidad e involucran la utilización de una mayor cantidad de insumos. Una de las chupeterías está empezando a fabricar chupetes con un valor de 0,2 soles. Estos están orientados al público escolar, por ello, se les denomina «chupete escolar».

La venta diaria de cada chupetería varía desde 200 hasta 3000 chupetes. El 75\% de ellas vende un mínimo de 1000 chupetes diarios. Todas las chupeterías venden el producto localmente. Dos de ellas, exclusivamente en Iquitos. Seis chupeterías (75\%) envían a Lima su producto; de estas, dos también lo hacen a Pucallpa.

El número total de trabajadores directos que emplea la industria del aguaje es de 41 personas. En las chupeterías con mayor capacidad de producción, el máximo número de vendedores es de cinco; el mínimo, de dos. Esta industria brinda trabajo a 189 personas como chupeteros; cada chupetero gana el $40 \%$ de las ventas realizadas y retorna los chupetes no vendidos a la fábrica.

En el caso de las bodegas que venden chupetes, estas los compran a la fábrica a un costo de 0,3 soles cada chupete y los venden a 0,5 soles al público. Estas asumen la pérdida en caso de no vender todos los chupetes. La heladería vende sus helados directamente al público. Esta solo otorga el $20 \%$ de ganancia sobre los chupetes que 
son vendidos por chupeteros. Por su parte, los heladeros venden en sus carritosheladeros solo en la ciudad. En este caso, el negocio es personal; los helados se ofertan desde de 0,5 soles hasta 1 sol. La venta diaria es de 100 helados; se vende más en la estación seca y en época escolar.

Tabla 3: Comercialización de helados y chupetes.

\begin{tabular}{|c|c|c|c|c|c|c|c|c|}
\hline \multicolumn{9}{|c|}{ Chupeterías y chupeteros } \\
\hline $\begin{array}{l}\mathrm{N}^{\circ} \\
\text { clave }\end{array}$ & $\begin{array}{l}\text { Sistema de } \\
\text { comercia- } \\
\text { lización }\end{array}$ & $\begin{array}{c}\text { Época } \\
\text { de mayor } \\
\text { venta }\end{array}$ & $\begin{array}{l}\text { Precio al } \\
\text { público en } \\
\text { soles }\end{array}$ & $\begin{array}{c}\mathrm{N}^{\circ} \text { de } \\
\text { chupetes } \\
\text { venta/diaria }\end{array}$ & $\begin{array}{c}\text { Venta en } \\
\text { otras } \\
\text { ciudades }\end{array}$ & $\begin{array}{c}\text { Trabaja- } \\
\text { dores } \\
\text { directos }\end{array}$ & $\begin{array}{c}\mathrm{N}^{\circ} \text { de } \\
\text { chupe- } \\
\text { teros }\end{array}$ & $\begin{array}{c}\text { Forma } \\
\text { de pago }\end{array}$ \\
\hline 1 & Bodegas & Verano & $0,5-1$ & 1000 & $\begin{array}{c}\text { Lima, } \\
\text { Pucallpa }\end{array}$ & 5 & no tiene & $40 \%$ \\
\hline 2 & Chupeteros & Verano & $0,5-1$ & $1000-1200$ & Lima & 5 & 70 & $40 \%$ \\
\hline 3 & Chupeteros & Verano & $0,5-1$ & 1000 & Lima & 5 & 50 & $40 \%$ \\
\hline 4 & $\begin{array}{l}\text { Chupeteros, } \\
\text { bodegas }\end{array}$ & $\begin{array}{l}\text { Verano, } \\
\text { fiestas }\end{array}$ & $0,5-1$ & $400-500$ & No & 2 & 15 & $40 \%$ \\
\hline 5 & $\begin{array}{l}\text { Chupeteros, } \\
\text { bodegas }\end{array}$ & Verano & $\begin{array}{c}0,2-0,5 \\
-1\end{array}$ & $2500-3000$ & $\begin{array}{l}\text { Lima, } \\
\text { Pucallpa }\end{array}$ & 4 & 6 & $40 \%$ \\
\hline 6 & En fábrica & Verano & 0,5 & $200-300$ & No & 3 & 3 & $40 \%$ \\
\hline 7 & Chupeteros & Verano & 0,5 & $1500-2000$ & Lima & 5 & 25 & $40 \%$ \\
\hline 8 & Chupeteros & Verano & 0,5 & 1000 & Lima & 3 & 10 & $40 \%$ \\
\hline \multicolumn{9}{|c|}{ Heladerías y heladeros } \\
\hline 1 & $\begin{array}{l}\text { En heladería } \\
\text { y chupeteros }\end{array}$ & Verano & $0,5-2$ & 1000 & No & 6 & 10 & $20 \%$ \\
\hline 2 & En puesto & Verano & $0,5-1$ & 100 & No & 1 & - & - \\
\hline 3 & En puesto & Verano & $0,5-1$ & 100 & No & 1 & - & - \\
\hline 4 & En puesto & Verano & $0,5-1$ & 100 & No & 1 & - & - \\
\hline
\end{tabular}

Verano o estación seca: junio-noviembre

1 dólar $=3,5$ soles

De la Tabla 3, tomando como base el precio de 0,5 soles por chupete, se puede deducir que la venta mínima diaria varía desde 100 hasta 1500 soles. Esta cifra, proyectada mensualmente, arroja entre 3000 y 45000 soles, respectivamente. Las cantidades proyectadas no son nada desdeñables para una empresa que se desarrolla en una economía de un país pobre como el Perú. Si se deducen los gastos operativos y las obligaciones tributarias, queda un saldo que permite vivir con comodidad a los propietarios. 


\section{4 Infraestructura, equipamiento, durabilidad de la masa e insumos utilizados}

Una chupetería es, básicamente, una casa en cuya sala se encuentra una congeladora en la que están colocados los chupetes para la venta al público. Asimismo, hay un ambiente en el que están ubicadas las demás congeladoras y la máquina chupetera, si es que se tiene. En otro ambiente, se almacenan los sacos de aguaje y se prepara la masa para los chupetes.

Según la Tabla 4, el número de congeladoras por chupetería varía desde dos, en las de menos producción, hasta 14 , en las de mayor producción. Las cinco $(62,5 \%)$ mayores chupeterías tienen como mínimo ocho congeladoras. Estas se usan para almacenar los chupetes producidos en las máquinas chupeteras.

En las máquinas, los moldes de chupetes llenos con la dilución de aguaje y demás insumos permanecen desde 5 hasta 30 minutos. Luego, estos son colocados en las congeladoras donde, según la cantidad de chupetes producidos y la forma de comercialización, pueden permanecer hasta tres días, pasados los cuales el producto comienza a perder el color y a volverse algo oscuro con lo cual pierde calidad.

La conservación de la masa es el punto crítico de la industria de transformación del aguaje, pues esta se oxida muy fácilmente y cambia del color amarillo que la caracteriza al negro, como máximo en tres días. Por esta razón, las chupeterías que producen una baja cantidad de productos solo mantienen la masa un día y las demás dos o tres días como máximo. Sin embargo, una de ellas logra mantener la masa hasta dos meses; para ello, no agrega ningún elemento a la masa.

Los heladeros solo utilizan la máquina heladera. Esta consta de una mesita rodante en cuyo costado hay una caja con hielo y sal. En ella, se coloca un cilindro metálico que contiene la masa de aguaje y los demás insumos. Para que el producto esté listo para venderse, el heladero solo necesita girar el cilindro en la caja por 1,5 horas. No es necesario almacenar el producto, pues lo que se procesa es lo necesario para la venta del día.

Los insumos básicos usados para la fabricación de los chupetes son: masa de aguaje, leche condensada o en polvo, azúcar, esencia de vainilla. En algunas chupeterías usan colorantes, saborizantes y estabilizadores. Los otros insumos y sus proporciones específicas usadas son el «secreto» de la chupetería. Con ellos, se marca la diferencia con respecto a los productos de las demás fábricas. 
Tabla 4: Equipos, congelación e insumos usados para elaborar chupetes y helados.

\begin{tabular}{|c|c|c|c|c|}
\hline \multicolumn{5}{|c|}{ Chupeterías } \\
\hline $\begin{array}{l}\mathrm{N}^{\circ} \\
\text { clave }\end{array}$ & $\begin{array}{c}\mathrm{N}^{\circ} \\
\text { congeladoras }\end{array}$ & $\begin{array}{l}\text { Tiempo de } \\
\text { congelación }\end{array}$ & $\begin{array}{l}\text { Duración de } \\
\text { la masa }\end{array}$ & Insumos \\
\hline 1 & 10 & N.I. & 1 día & N.I. \\
\hline 2 & 9 & 10 horas & 1 día & $\begin{array}{l}\text { Aguaje, leche, azúcar, } \\
\text { vainilla }\end{array}$ \\
\hline 3 & 8 & 10 horas & 1 día & $\begin{array}{l}\text { Aguaje, leche, azúcar, } \\
\text { vainilla }\end{array}$ \\
\hline 4 & 4 & $\begin{array}{l}30 \text { ', luego a la } \\
\text { conservadora }\end{array}$ & 2 - 3 días & $\begin{array}{l}\text { Aguaje, leche, vainilla, } \\
\text { azúcar, colorantes }\end{array}$ \\
\hline 5 & 14 & 3 días & $\begin{array}{l}2 \text { meses } \\
\text { sin azúcar } \\
\text { ni leche }\end{array}$ & $\begin{array}{l}\text { Aguaje, leche, azúcar, } \\
\text { esencia }\end{array}$ \\
\hline 6 & 2 & $\begin{array}{l}20 ', \text { luego a la } \\
\text { conservadora }\end{array}$ & 2 días & $\begin{array}{l}\text { Aguaje, leche, azúcar, } \\
\text { vainilla }\end{array}$ \\
\hline 7 & 11 & 2 días & 2 días & $\begin{array}{l}\text { Aguaje, leche, azúcar, } \\
\text { vainilla, saborizante }\end{array}$ \\
\hline 8 & 5 & $\begin{array}{l}\text { 5', luego } 30 \text { ' en la } \\
\text { congeladora }\end{array}$ & 2 días & $\begin{array}{l}\text { Aguaje, leche, azúcar, } \\
\text { vainilla, colorante, } \\
\text { estabilizador }\end{array}$ \\
\hline \multicolumn{5}{|c|}{ Heladerías y heladeros } \\
\hline 1 & 3 & $\begin{array}{c}\text { Helados 10' } \\
\text { Chupetes } 2 \text { horas }\end{array}$ & 1 día & N.I. \\
\hline 2 & 1 Máquina & 1,5 horas & 1 día & Aguaje, azúcar \\
\hline 3 & 1 Máquina & 1,5 horas & 1 día & Aguaje, azúcar \\
\hline 4 & 1 Máquina & 1,5 horas & 1 día & Aguaje, azúcar \\
\hline
\end{tabular}

N.I. = no informó.

\subsection{Tecnología de transformación de chupetes y helados}

El proceso para elaborar chupetes supone, básicamente, el proceso que se describe a continuación. Después de lavados los frutos del aguaje, estos son colocados en un recipiente donde se agrega agua caliente. Con esta agua, los frutos permanecen por espacio de un día. Cuando los frutos están suaves, se colocan en un recipiente de madera donde son machacados hasta que desprendan sus semillas. Luego, se eliminan las semillas que queden y la cáscara. A la masa sobrante, se le agrega agua 
gradualmente hasta obtener la dilución deseada. Después, dicha masa se cierne para eliminar las cascarillas remanentes. A continuación, se agrega leche, vainilla y azúcar. Finalmente, se vacía el preparado en los moldes, que se colocan en la máquina chupetera o en la congeladora.

En el caso de la chupetería Shambo, que no compra masa sino dilución, solo se eliminan los pasos de ablandamiento, despulpado y cernido. Salvo lo indicado, todo el proceso es similar.

El proceso para la elaboración del helado es mucho más simple y se realiza como a continuación se describe. A la masa de aguaje se le agrega agua. Se bate o «chapea» hasta que la masa se diluya. Luego, se cierne la masa para eliminar la cáscara de los frutos. Se agrega azúcar y luego se coloca el preparado en un recipiente de acero inoxidable que está inmerso en una caja que contiene hielo y sal. Allí, el recipiente metálico es girado por un espacio de 1,5 horas, aproximadamente. Durante este lapso, se congela la masa que pronto estará lista para la venta al público.

\subsection{Perspectivas de los industriales sobre el aguaje}

El 50\% de los propietarios de las chupeterías considera que la universidad local debe orientar sus esfuerzos para encontrar técnicas que permitan conservar la pulpa del aguaje. El otro $50 \%$ opina que la universidad debe desarrollar o proponer equipos mecánicos para facilitar el proceso de producción. Tal sería el caso de aparatos despulpadores, separadores de pulpa y cáscara, eliminadores de semillas, que permitan lavar y pasteurizar la pulpa, conservar el sabor de la pulpa, etc. Los propietarios también esperan propuestas de cómo mejorar la calidad del producto a través del marketing y los sistemas de venta.

El 75\% de los industriales opina que está consciente de que las palmeras de aguaje están cada vez más escasas debido a la forma de extracción (corte de la planta). El $25 \%$ considera que la oferta de los frutos siempre existió y existirá y no encuentra mayores problemas en continuar produciendo.

Sin embargo, son los industriales más antiguos y con mayor capacidad de transformación los que opinan que se debe hacer algo para evitar el corte de los aguajes. Esta actitud positiva de la mayoría de los industriales puede permitir concertar políticas para lograr el aprovechamiento sostenible de la especie. La concertación debería ser entre las diversas instituciones públicas y privadas que están relacionadas con esta especie: las universidades, los institutos de investigación, las organizaciones ambientales, los productores, los comerciantes, los industriales y el Estado. 


\section{BIBLIOGRAFÍA}

BALICK, M. J. 1979. Amazonian oil palms of promise: a survey. En: Economic Botany 33(1) 11-28. The New York Botanical Garden.

1982. Palmas neotropicales: nuevas fuentes de aceites comestibles. En: Interciencias, 7 (1): 25-29.

BRAUN, A. 1968. Cultivated palms of Venezuela. En: Principes 12(2,3,4).

FLORES, P. S.; GÓMEZ, R. E.; KALLIOLA, R. 1998. Características generales de la zona de Iquitos. En: Kalliola, R; Flores, P. S. (ed). Geoecología y desarrollo amazónico, estudio integrado en la zona de Iquitos, Perú. Turku (Finlandia): Turunyliopisto. $544 \mathrm{pp}$.

GRANVILLE, J. J. 1974. Apercu sur la structure des pneumatophores de deux espeses des sols hydromorphes en Guyane. Cah. ORSTOM, Ser. Biol. $\mathrm{N}^{\circ} 23$ : 3-22.

HEINEN, H. D.; RUDDLE, K. 1974. Ecology, ritual and economic organization in the distribution of palm starch among the Warao of the Orinoco delta. En: Journal of Anthropologic Research 30:116-138.

HENDERSON, A. 1995. The palms of the Amazon. New York: Oxford University Press. 361 pp.

HENDERSON, A.; GALEANO, G.; BERNAL, R. 1995. Field guide to the palms of the Americas. New Jersey (USA): Princeton University Press. 352 pp.

HIRAOKA, M. 1999. Miriti (Mauritia flexuosa) Palms and their uses and management among the ribeirinhos of the Amazon Estuary. In: Padoch, C.; Ayres J. M.; Pinedo-Vásquez, M.; Henderson, A. (ed). Varzea, diversity, development, and conservation of amazonas wtithewater floodplains. The New York Botanical Garden. 407 pp.

INEI. 1993. Resultados definitivos de los censos nacionales IX de población, IV de Vivienda y I de comunidades nativas. Perú. 
MARENGO, J. A. 1998. Climatología de la zona de Iquitos, Perú. En: Kalliola, R; Flores P. S. (ed.). Geoecología y desarrollo amazónico, estudio integrado en la zona de Iquitos, Perú. Turku (Finlandia): Turunyliopisto. 544 pp.

NATIONAL ACADEMY OF SCIENCES (NAS). 1975. Underexploited tropical plants with promising economic value. Washington D.C. 189 pp.

PADOCH, C. 1988 a. The economic importance and marketing of forests and fallow products in the Iquitos region in Swidden-fallow agroforestry in the Peruvian Amazon. En: Denevan, W.M.; Padoch, C. (ed.). Advances in Economic Botany 5. New York Botanical Garden, Bronx. pp 74-89.

1988 b. People of the foodplain and forest. En: Denslow, J. S.; Padoch, C. (ed.). People of the tropical forest. Berkeley: University of California Press. pp 127-14.

1992. Marketing of non-timber forest products in western Amazonia: General observations and research priorities. En: Advances in Economic Botan. 9: 43-50. New York.

ROJAS, R. R. 1985. Ensayos de germinación con semillas de 5 especies de palmeras aplicando 10 tratamientos pre-germinativos y ensayos de cosecha con 7 métodos. Iquitos (Perú): Universidad Nacional de la Amazonía Peruana. Tesis de Ingeniero Forestal. $110 \mathrm{pp}$.

RUIZ, M. J. 1991. El aguaje, alimento del bosque amazónico. En: Temas forestales N 8. Pucallpa (Perú): Cotesu. 28 pp.

RUIZ, M. J. 1993. Alimentos del bosque amazónico: una alternativa para la protección de los bosques tropicales. Montevideo (Uruguay): UNESCO/ORCYT. $225 \mathrm{pp}$.

VICKERS, W. T. 1976. Cultural adaptation to Amazonian habitats: The Siona-Secoya of Easter Ecuador. Gainesville (USA): University of Florida, Tesis Ph. D. 